Regular Paper

\title{
Measurement of sEMG and hand motion identification for hand rehabilitation equipment development
}

\author{
Misato NIREI(Stu. Mem.) ${ }^{* 1}$, Masaya WATADA(Mem.) ${ }^{* 1}$ Mutsuo YAMADA*2 $^{*}$
}

In recent years, the population of disabled people has been increasing tendency in Japan. Among them, physical disability due to hemiplegia is the most common. For hemiplegic patients to live independent living, grasp and pinch motions are necessary. To realize this movement, it is necessary to move the first finger and the fifth finger separately. The purpose of my research was to development of hand rehabilitation equipment which for enables hemiplegic patients independent movements of first finger and fifth finger at the home. In this paper, first we propose rehabilitation equipment. Then, we investigate whether we can use the feature values of sEMG obtained from the forearm as a control signal for the proposed rehabilitation equipment. As a result, we considered that the discrimination result of each motion were insufficient in the evaluation using the threshold. Furthermore, the discrimination rate of flexion motion and extension motion by SVM was $64 \%$. In the future, we will make measurements at several places for improve accuracy. Based on it, we consider effective electrodes position.

Keywords: hemiplegia, rehabilitation, hands, sEMG, motion discrimination

(Received: 20 July 2018, Revised: 26 December 2018)

\section{Introduction}

In recent years, the population of disabled people has been increasing tendency in Japan. Among them, the most common is limbs inconvenience. The main cause of limbs inconvenience is hemiplegia due to cerebrovascular disorder. Hemiplegia refers to a state in which the tension of muscles on one side is extremely increased. This causes problems in everyday activities (meals, organizing, cleaning, etc.). In daily life, movement of the upper limbs is necessary. Therefore, improvement of upper limb movement is important for improving quality of life (QOL). Rehabilitation is important for improving behavior. Especially, hands are more prone to muscle atrophy than shoulders and elbows, and joints are easy to stiff. Therefore, continuous rehabilitation is necessary. In this research, we focused on hands.

Hemiplegic patients to live independent living, the grasp and pinch motions are required. To realize these motions, need to be able to motion first finger and fifth finger separately. As an opinion of an actual hemiplegic patient, it is cumbersome to go to a hospital for rehabilitation. However, the equipment which can independently practice each fingers is big and has been unsuitable for home use. The purpose of my research was to development of hand rehabilitation equipment which for enables hemiplegic patients independent movements of first finger and fifth finger at the home.

\footnotetext{
Correspondence: Misato NIREI, major in Biomedical Engineering, Tokyo City University, 1-4-6 Tamazutsumi, Setagaya-ku, Tokyo 158-8557, Japan email: g1781421@tcu.ac.jp

${ }^{* 1}$ Tokyo City University ${ }^{* 2}$ Ryutsu Keizai University
}

In this paper, first we propose rehabilitation equipment.

Then, we describe measurement method and processing method of the surface electromyogram (sEMG) as using for the equipment control. Finally, we describe result of the experiment and its consideration.

\section{Proposal of rehabilitation equipment}

\subsection{Necessity of rehabilitation equipment}

Hemiplegic patients are difficult to realize the same physical function as before onset of hemiplegia. Hemiplegia patients compensate for the lost physical function by undertake rehabilitation.

There are two approaches for the purpose of to undertake rehabilitation.

Approach to muscle: Because of many hemiplegic patients onset spastic paralysis, hemiplegic patients are difficult to move their hand. When the wrist joint does not move, joint contracture (limitation of the joint motion range) occurs. To prevent contractures, move hands passively is necessary.

Approach to the brain: The cerebrovascular disorder remains disorder the physical function of the site damaged. However, due to rehabilitation, compensatory mechanism by other parts of the brain, or some physical functions be improved are expected. For this purpose, voluntary exercise and the motion training intended by the patient him/herself is important [1].

\subsection{Concept of proposed equipment}

From 2.1, the aim of this research was to the development of rehabilitation equipment that practicing the movement for the paralyzed side's hand in the state of have an image to move by the intention of him/herself. Also, for an independent life, motions such as grasp and pinch are very important. For stable grasp 
and pinch motions are needed independent motion of the first finger and fifth finger. In addition, hemiplegic patients adopt a posture that grips hand by spastic paralysis. Therefore, to practice of flexion and extension motions are necessary. From this, we focused on the following 10 motions, and we proposed rehabilitation equipment that realizes these motion exercises.

- All finger, first finger, fifth finger, second $\sim$ fourth finger, a total of 4 types of flexion and extension $(8$ motion)

- First - fifth finger, first - second $\sim$ fourth finger total of 2 kinds each pinch ( 2 motion)

We proposed rehabilitation equipment that satisfies the above.

Fig. 1 shows the schematic diagram of the proposed rehabilitation system.

In the proposed rehabilitation equipment, reading the signals related to each operation from the hand on the healthy side. On the basis of these signal, the hand on the paralyzed side making the same movement as the hand on the healthy side by actuator. We expected by moving both hands, to be image training to move the hand on the paralyzed side by him/herself. In addition, rehabilitation is important to do continuously. Therefore, we required to do be able to use the rehabilitation equipment continuously in the home. For that purpose, it was necessary that even one hemiplegic patient can be used. Therefore, we considered the shape of equipment that a hemiplegic patient can be worn without assistance. This research's target person was a hemiplegia patient who has no joint contracture and was capable of general rehabilitation.

\section{Consideration of control signal in proposed equipment}

\subsection{About the control signal to be used}

As the concept of the proposed equipment, we cited the shape of the master which is easy to wear by hemiplegic patients. In the case of the glove type, detailed work of attaching to each finger is required. Therefore, it is difficult to wear by the hand on the paralyzed side. From this, master's shape was desirable to have a simple one as not be worn on fingers.

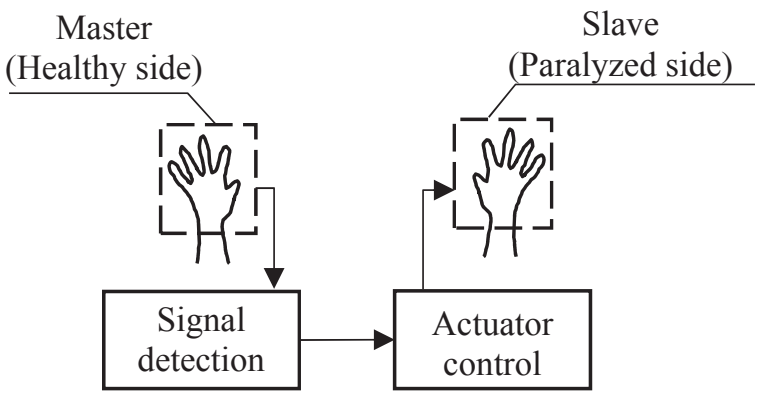

Master: Read signal of related to finger motion

Slave: Follows the movement of the Master

Fig. 1 Schematic diagram of the proposed rehabilitation system [2].
Among biological signals that can be measured from the body, sEMG can be measured from the body surface. Also, the muscles involved in flexion and extension of the fingers are running on the forearm. We believed to realize a master with a simple shape is possible if we can acquire signals necessary for motion recognition of each finger from the forearm surface. Therefore, we proposed to use sEMG as a control signal.

\subsection{Measurement experiment of sEMG related to flexion and extension motion}

In a previous research by Mr. Kikuchi, it was possible to distinguish the motion of the flexion and extension meter 6 other than the first finger and the first finger the feature value obtained from the measured sEMG arranging four channels of surface electrodes on the forearm part by using the discriminator [3].

In this research, first, we got the feature value of the sEMG obtained from the forearm part. Then, we examined whether it is effective for identifying the 10 motions mentioned in the concept. Finally, we considered whether it can be used as a control signal. We used integrated electromyogram (IEMG) the same as Mr. Kikuchi's method for the feature value in this research. IEMG is regarded as an index reflecting the whole activity state of the muscle, and becomes stronger as the muscle contraction is stronger. The reason for using IEMG, higher discrimination rate can be obtained when using support vector machine (SVM) which is a discriminator that can obtain a relatively high discrimination rate [4].

\subsubsection{Determination of electrode position}

The measurement position of the sEMG was decided with reference to anatomy [5]. Fig. 2 shows the electrodes position. The extensor pollicis brevis muscle of $\mathrm{Ch} 1$ and the flexor pollicis longus muscle of $\mathrm{Ch} 3$ and the abductor pollicis longus muscle of $\mathrm{Ch} 2$ assumed extension and flexion of the first finger. The flexor digitorum superficialis muscle of the $\mathrm{Ch} 4 \cdot$ the extensor digitorum muscle of $\mathrm{Ch} 7$ assumed flexion and extension of second $\sim$ fourth finger. The fifth finger has no flexor muscle. Therefore, it was decided on the assumption that the flexor carpi ulnaris muscle of Ch5 and the extensor carpi ulnaris of Ch6 correspond to flexion / extension of fifth finger.

Fig. 3 shows the arm cover that we used to record the electrode position. We drew a line at elbow and the base of wrist. Also, the center line was drawn so as to come to the position of the third finger, and a hole was drilled at the position where the electrode was actually affixed. As a result, we thought that reproducibility could be expected when conducting the experiment a plurality of times by putting the electrodes again. 


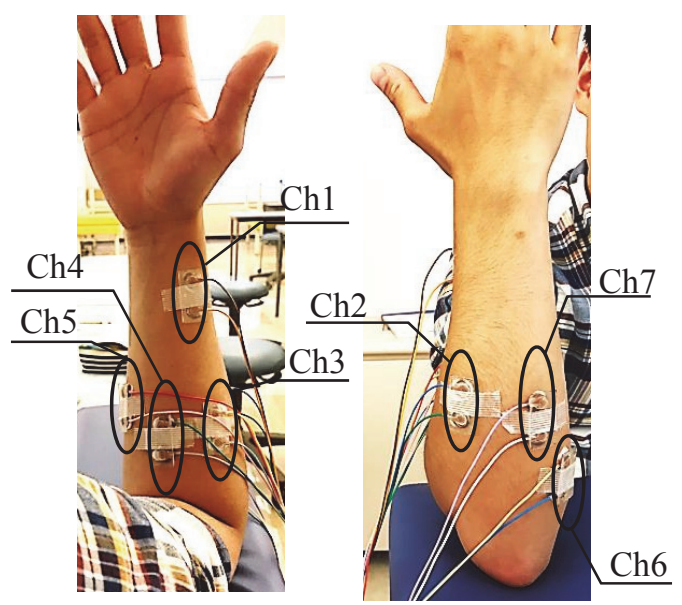

Fig.2 Electrodes position.

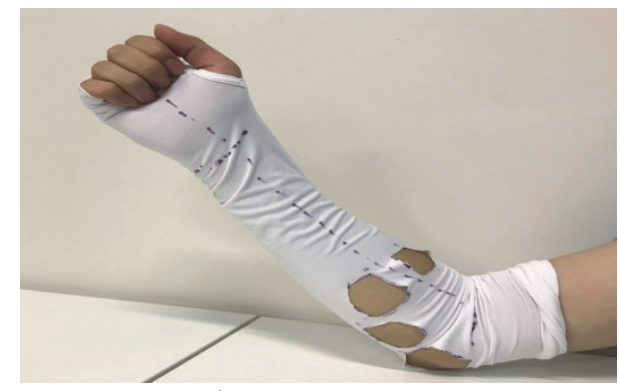

Fig. 3 Arm cover.

\subsubsection{Method of fixing electrodes}

The forearm is a curved surface, the electrode is easy to peel. For this reason, we used the dish electrodes for brain waves (Nihon Kohden Corporation, NE-113A). Fig. 4 shows electrodes used the measurement. After disinfecting the body surface with alcohol, we pasted almost parallel to the muscle fiber by electrocardiogram paste (Nihon Kohden Corporation, cardioCream Z101BC) as shown in Fig.2. At this time, the wrist side was arranged to be + on all channels. In addition, the electrodes were fixed by winding a rubber sheet from the top secured with a surgical tape. Fig. 5 shows the method of fixing electrodes.

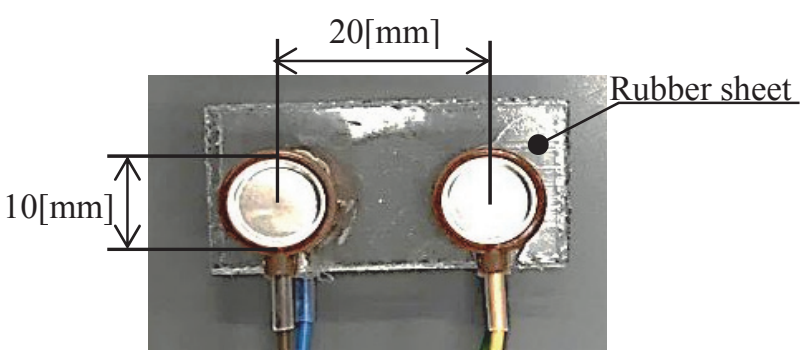

Fig. 4 Electrodes.

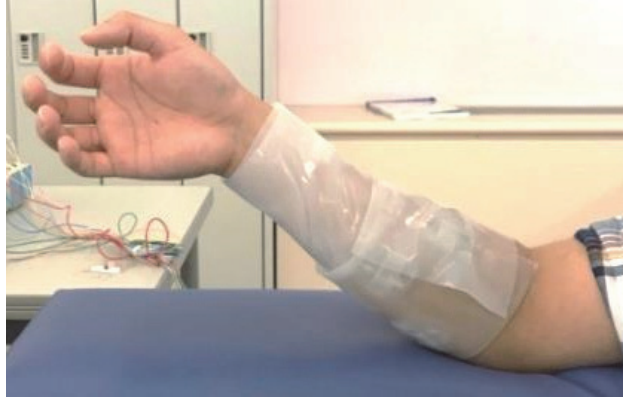

Fig. 5 Method of fixing electrodes.

\subsubsection{Experimental method}

We carried out the experiment with two healthy men (23, 24 years old). Fig. 6 shows the connection of the device, and Table 1 shows the setting of the biological amplifier. We measured sEMG at sampling frequency $1000 \mathrm{~Hz}$ and amplified to 500 fold with a biological amplifier (Nihon Kohden Corporation, Dual-Channel Bioelectric Amplifier MEG-5200). The hand motions in measurement is shown in Fig. 7.

We defined motion A, B was flexion and extension of all fingers, Motion $\mathrm{C} \sim \mathrm{E}$ was extension of each finger, Motion $\mathrm{F} \sim \mathrm{H}$ was the flexion of each finger, Motion I and $\mathrm{J}$ was a pinch motion.

Fig. 8 illustrates protocol of the measurement. One set of the measurement time was 20 seconds. The measurement started from Motion X (state where no force was put on hand). Subjects performed the operation designated one of Motion A - J, intervals of 3 seconds four times. At this time, we set to operate one second by timing of Motion. We performed the measurement each 15 times for Motion A - J. In addition, the order of the measurement Motion was performed as follows.

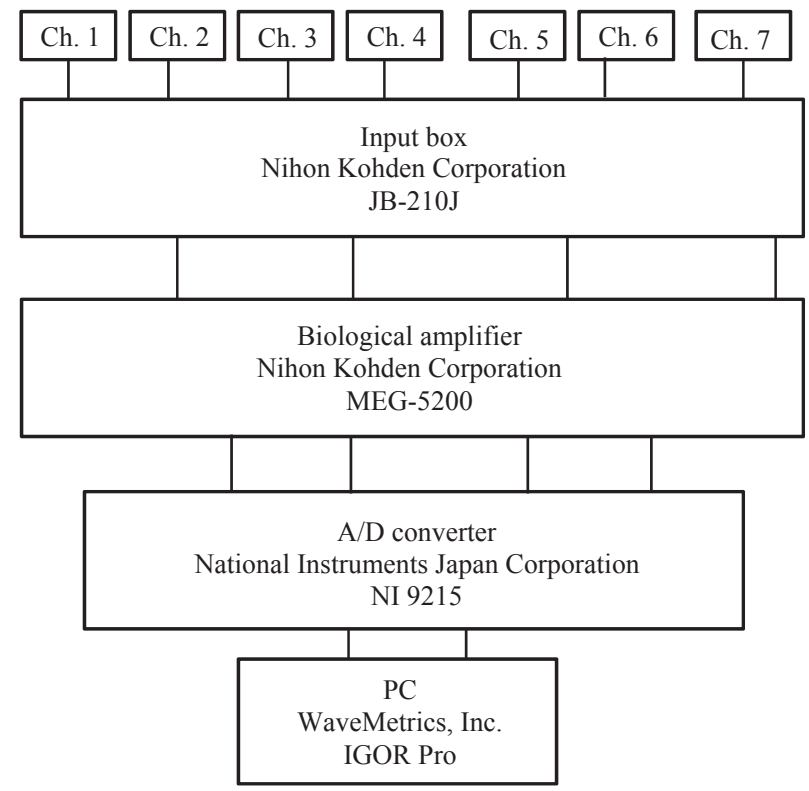

Fig. 6 Connection of the device. 
Table1 Setting of the biological amplifier.

\begin{tabular}{|c|c|c|c|}
\hline GAIN & HUM FIL & LO CUT[Hz] & HI CUT[Hz] \\
\hline 500 & ON & 150 & 3000 \\
\hline
\end{tabular}

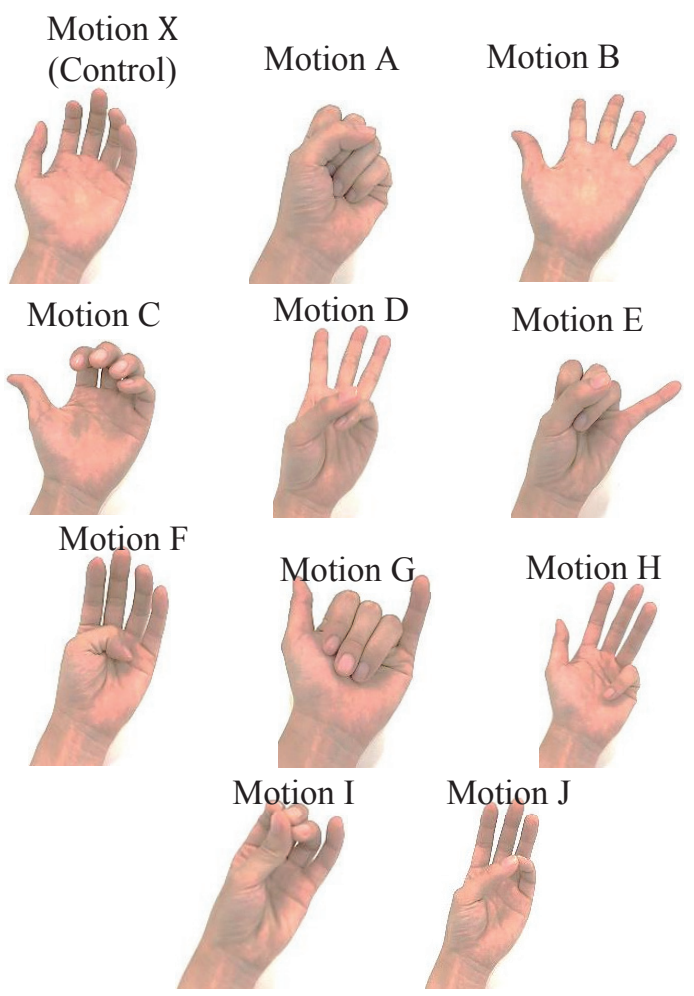

Fig. 7 Hand motions in measurement.

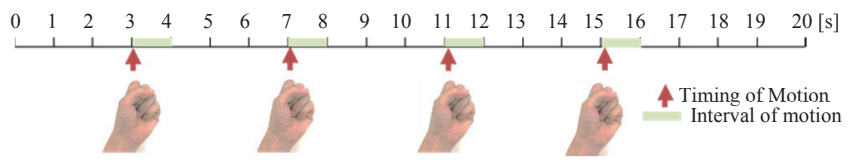

Fig. 8 Protocol of the measurement.

$$
\mathrm{A} \rightarrow \mathrm{B} \rightarrow \mathrm{F} \rightarrow \mathrm{C} \rightarrow \mathrm{G} \rightarrow \mathrm{D} \rightarrow \mathrm{H} \rightarrow \mathrm{E} \rightarrow \mathrm{I} \rightarrow \mathrm{J}
$$

In order not to be affected by similar movements by previous and next measurements, we made the order of Motion random. The time from the completion of the measurement to the next one was set to 1 minute or more.

At the time of measurement, the subject was seated in the chair and the right arm elbow was put on the elbow pillow. The elbow angle was $90^{\circ}$ and the palm kept sideways (the first finger was on the ceiling side).

We measured sEMG under the above conditions, and performed on Full-wave rectification. Next, performed smoothing by a Butterworth filter (cutoff frequency of $2.5 \mathrm{~Hz}$ ). After that, we acquired IEMG by down sampling to $200 \mathrm{~Hz}$ in order to reduce the data volume. As an example, Fig. 9 illustrates the IEMG obtained in Motion A (1st set).

Further, we obtained sum of the IEMGs. For example, Fig. 10 illustrates sum of the IEMGs in Fig. 9.

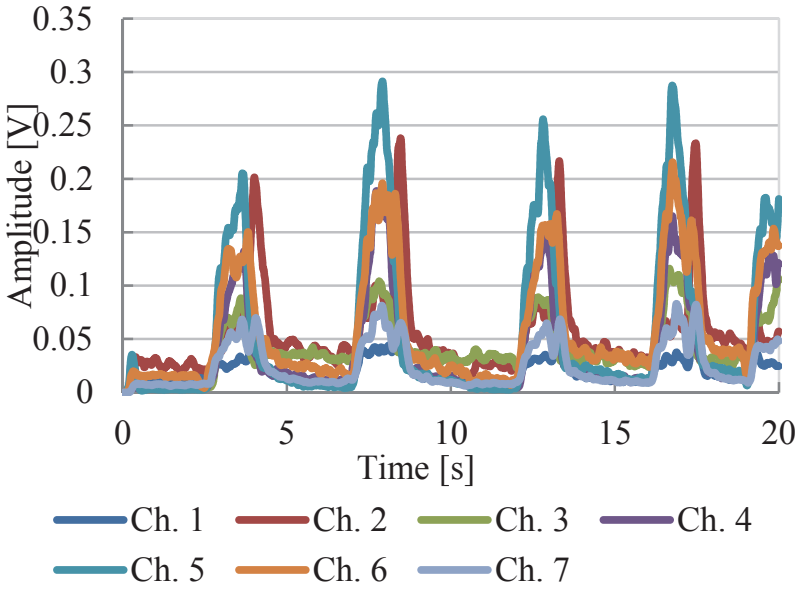

Fig. 9 IEMG obtained in Motion A (subject A, 1st set).

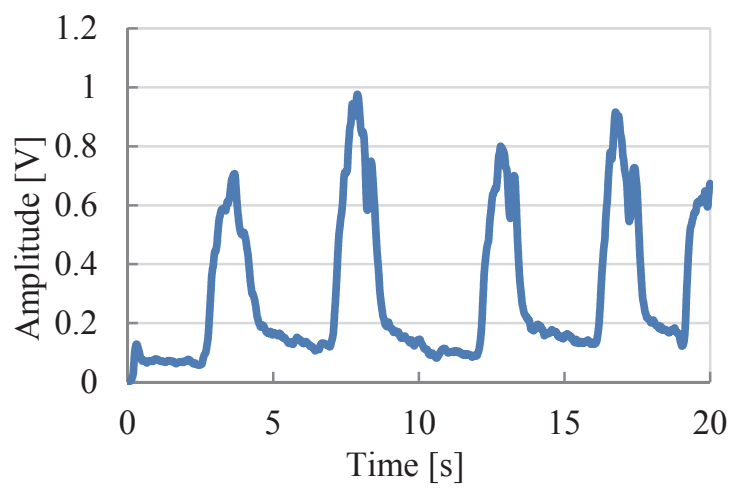

Fig. 10 Sum of the IEMGs in Fig. 9.

We obtained the maximum value at each timing motion from sum of the IEMGs. In this research, we treated the value of the extracted each channel at that plotted point as a feature values.

In this experiment, as a result evaluation method, we decided that it was operating when $70 \%$ or more exceeds the threshold value. The threshold value set 0.1 $\mathrm{V}$.

Moreover, in order to investigate whether it was a feature values effective for motion discrimination, we prepared and evaluated SVM which discriminates flexion and extension motion in a simply [6]. SVM program was created using python 3.6. The data set consists of totaling 600 times (10 motions by 15 sets). Training data was divided into $70 \%$ and test data $30 \%$. We set flexion (including pinch motion) at 1 and extension at -1 , and we confirmed the correct answer rate for the test data.

\subsection{Experimental Results and Discussion}

As an example, Fig. 11 and Fig. 12 illustrate the feature values obtained in Motion A by subject A and B. In addition, Tables 2, 3 show the results of adapting the threshold values to the feature values in each motion. 


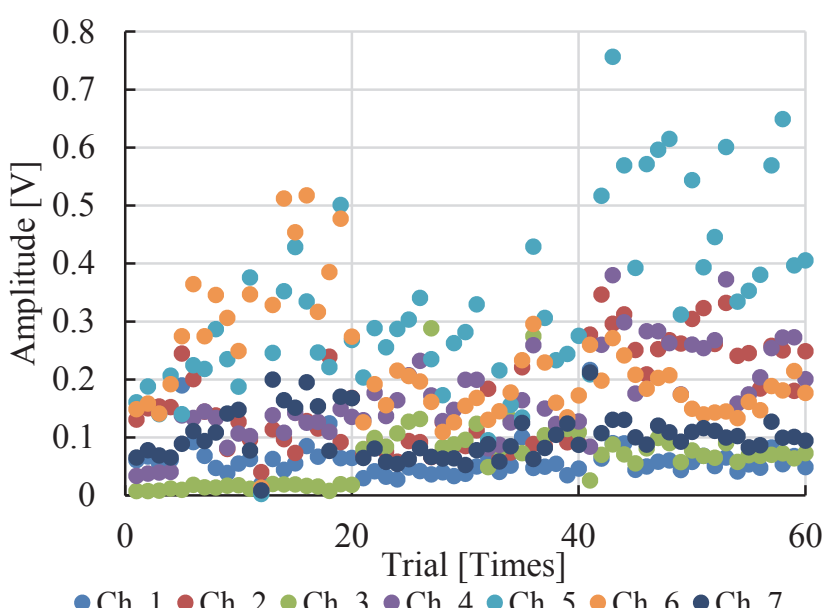

Fig. 11 Feature values obtained in Motion A (subject A).

Table2 Results of adapting the threshold values to the feature values (Subject A).

\begin{tabular}{|c|c|c|c|c|c|c|c|}
\hline Motion & Ch. 1 & Ch. 2 & Ch. 3 & Ch. 4 & Ch. 5 & Ch. 6 & Ch. 7 \\
\hline A & & & & $\bullet$ & $\bullet$ & $\bullet$ & \\
\hline B & $\bullet$ & $\bullet$ & & & $\bullet$ & $\bullet$ & $\bullet$ \\
\hline C & $\bullet$ & $\bullet$ & & & & $\bullet$ & $\bullet$ \\
\hline D & & $\bullet$ & & & & & \\
\hline E & & $\bullet$ & & & & & \\
\hline F & & $\bullet$ & & & & & \\
\hline G & & & & $\bullet$ & $\bullet$ & $\bullet$ & \\
\hline H & & $\bullet$ & & & & & \\
\hline I & & $\bullet$ & & & $\bullet$ & & \\
\hline J & & $\bullet$ & & & & & $\bullet$ \\
\hline
\end{tabular}

The shaded part of the table is to correspond we assumed muscle activity at motions. $\bullet$ is the place that judged it was operating. From the tables, there was not much correspondence with the muscle activity it was assuming in each motion. Regarding Ch. 5 and Ch. 6, originally, the place of the muscle that involve in dorsal flexion / ulnar flexion of the wrist joint. Therefore, they are influenced by the motion of the wrist joint. In Motion A and B, we considered the muscles worked greatly in order to move the entire hand when performing the motion. In addition, the abductor pollicis longus muscle of Ch. 2 is a deep muscle, and the extensor digitorum muscle in the surface layer. It is also the muscle which is also involved in radial flexion of the wrist joint. Therefore, we considered that active activities were seen in multiple motions.

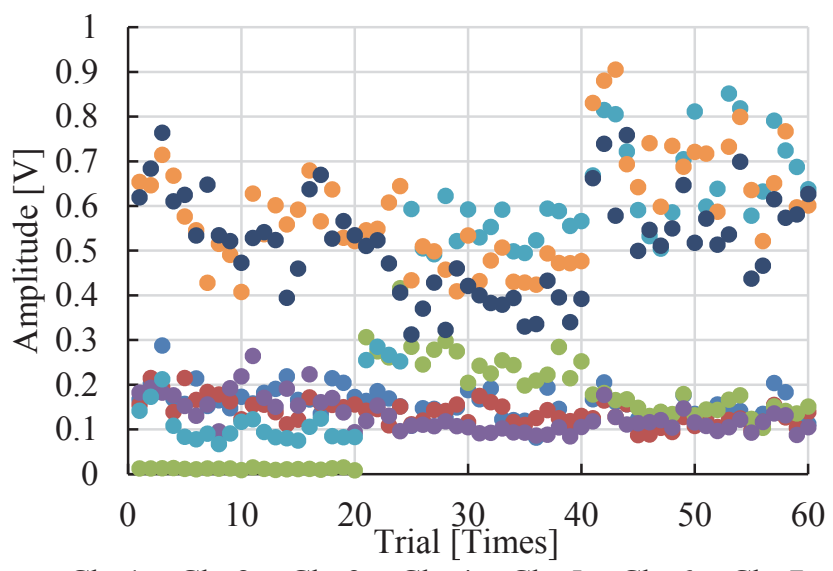

$\bullet$ Ch. $1 \bullet$ Ch. $2 \bullet$ Ch. $3 \bullet$ Ch. $4 \bullet$ Ch. $5 \bullet$ Ch. $6 \bullet$ Ch. 7

Fig. 12 Feature values obtained in Motion A (subject B).

Table3 Results of adapting the threshold values to the feature values (Subject B).

\begin{tabular}{|c|c|c|c|c|c|c|c|}
\hline Motion & Ch. 1 & Ch. 2 & Ch. 3 & Ch. 4 & Ch. 5 & Ch. 6 & Ch. 7 \\
\hline A & $\bullet$ & $\bullet$ & & $\bullet$ & $\bullet$ & $\bullet$ & $\bullet$ \\
\hline B & $\bullet$ & $\bullet$ & & & & $\bullet$ & \\
\hline C & $\bullet$ & $\bullet$ & & & & & $\bullet$ \\
\hline D & & $\bullet$ & & & & & \\
\hline E & $\bullet$ & $\bullet$ & & & & & \\
\hline F & & & & & & & \\
\hline G & & $\bullet$ & & & & & \\
\hline H & & & & & & & \\
\hline I & & $\bullet$ & & & & & \\
\hline J & & $\bullet$ & & & & & $\bullet$ \\
\hline
\end{tabular}

Also, no extreme difference was observed for each subject. However, subject B had fewer places where it was judged operating compared to subject A.

Next, as a result of identifying flexion and extension motions using SVM, the result was $74 \%$ for Subject A and $64 \%$ for Subject B. In the previous research by Mr. Kikuchi and Mr. Inui et al. Using SVM, the result that the discrimination rate is $80 \%$ or more has been obtained $[3,4]$. Therefore, we considered these number was to be low. We consider that the result is related to the value of the parameter. In the SVM used in this experiment, the RBF kernel was used as a kernel function. Further, the value $g$ of the parameter in the initial setting was $g=0.001$. In the RBF kernel, it has been clarified that high discrimination rate can be obtained by setting $g=0.5-1[4]$. Therefore, also in this study, we believe that accuracy can be improved by adjusting parameter values. In addition, discrimination rate of subject B was 10\% lower than A. As a cause of this, from the table, we considered that the discrimination rate was low because noticeable 
differences are not seen in multiple motions. Also, for channels that were not seen much activity, we believed that less trials were involved. This time, the number of trials of one Motion was as small as 60 in totals. We consider that accuracy improvement will be able to make by increasing the number of trials.

\section{Conclusion}

In this research, first we proposed rehabilitation equipment that can be used even by a single hemiplegic patient in the home. Then, we obtained the feature values from IEMG for 7 channels. After, we investigated whether each motion can be distinguished by feature values. In addition, we investigated whether flexion and extension motion can be identified by using SVM. However, discrimination of each motion by evaluation using a threshold value was insufficient. In addition, the discrimination of flexion and extension motion by SVM resulted in a low result of $64 \%$. Therefore, in this result, we considered that it is insufficient for use as a control signal. In the future, we will conduct measurements at several places and investigate effective electrode locations for improve the accuracy. In addition, we will construct a classifier and consider whether it is possible to identify the 10 motions mentioned in the concept. After that, we will propose measuring equipment that can be worn with one hand, consider fabrication, evaluation, taking into consideration posture at the time of use and characteristics at individuals.

\section{References}

[1] I. Isao, "Undoukinoukaihuku wo mokutekitosita nousottyu-lihabilitation no noukagaku wo konkyotosuru rironn to sonozissai," Medical Journal of Aizawa Hospital, Vol.8, pp4, 2010, (in Japanese).

[2] M. Nirei, M. Masaya, M. Yamada, "Proposal of hand rehabilitation device for home-piece paralyzed patients that can be used alone and examination of control signals", proceedings of the $26^{\text {th }}$ MAGDA Conference, pp.376, Kanazawa, Oct. 2017.

[3] T. Kikuchi, "Identification of finger operation using support vector machine and control of myoelectric prosthetic hand based on integrated electromyogram," in proceedings of the 2014 IEEE International Conference on Robotics and Biomimetics, pp.1273-1274, Bali, Dec. 2015.

[4] D. Inui, S. Ito, M. Sasaki, "Experimental Considerations on Signal Feature and Kernel/Parameters of SVM in Hand Motion Classification from sEMG," Transactions of the Japan society of mechanical engineers. C., vol.79, No.808, pp.4748-4750, 2013, (in Japanese).

[5] Y. Ueba, "Te sono kinou to kaibou", kinpodo, Japan, 2010.

[6] https://algorithm.joho.info/machine-learning/pythonscikit-learn-svm-training/,(cited2018-7-11),(in Japanese). 